\title{
Multilinguales
}

\section{Les grandes tendances de l'alternance des langues dans la presse écrite d'Algérie}

The Main Tendencies of Language Alternation in Algerian Newspapers

\section{Mourad BEKTACHE}

\section{OpenEdition}

1 Journals

Édition électronique

URL : http://journals.openedition.org/multilinguales/2468

DOI : $10.4000 /$ multilinguales. 2468

ISSN : 2335-1853

Éditeur

Université Abderrahmane Mira - Bejaia

\section{Édition imprimée}

Date de publication : 1 décembre 2013

Pagination : 91-105

ISSN : 2335-1535

\section{Référence électronique}

Mourad BEKTACHE, « Les grandes tendances de l'alternance des langues dans la presse écrite d'Algérie », Multilinguales [En ligne], 2 | 2013, mis en ligne le 01 décembre 2013, consulté le 17 septembre 2019. URL : http://journals.openedition.org/multilinguales/2468 ; DOI : 10.4000/ multilinguales. 2468

Ce document a été généré automatiquement le 17 septembre 2019.

\section{cc) $(1) \ominus$}

Multilinguales est mise à disposition selon les termes de la Licence Creative Commons Attribution -

Pas d'Utilisation Commerciale - Pas de Modification 4.0 International 


\section{Les grandes tendances de l'alternance des langues dans la presse écrite d'Algérie}

The Main Tendencies of Language Alternation in Algerian Newspapers

Mourad BEKTACHE

\section{NOTE DE L'ÉDITEUR}

Cet article a déjà été publié, sous le même titre, dans Multilinguales $\mathrm{n}^{\circ} 1$, mais délesté malencontreusement de quelques pages, pour des raisons purement techniques. C'est pour le rétablir dans sa version complète qu'il est repris ici.

1 La variation du français employé en Algérie ne se limite pas aux créations lexicales, aux emprunts et aux changements sémantiques. Elle se manifeste également à travers l'alternance et le mélange de langues. Les Algériens alternent et mélangent plusieurs langues: l'arabe dialectal, l'arabe standard, les variétés du berbère, le français et accessoirement l'anglais. Ce phénomène linguistique, très caractéristique de l'oral, apparaît aussi à l'écrit : il concerne, à titre d'illustration, les romans, les revues et la presse écrite.

2 Dans le cadre de cette étude, nous nous attacherons à décrire et à expliquer les alternances de langues, en nous intéressant particulièrement au phénomène de la polyphonie qui caractérise les alternances de langues dans le discours journalistique francophone algérien.

3 L'analyse des grandes tendances des alternances codiques relevées dans notre corpus permet de comprendre l'emploi des langues en Algérie (arabe standard, arabe dialectal et berbère). En effet, dans la presse écrite algérienne d'expression française, l'alternance et le mélange de langues apparaissent comme des procédés linguistiques de communication à travers lesquels se traduit le type de rapport que les langues en 
contact entretiennent. Cette alternance et/ou mélange de langues s'inscrit dans le cadre d'une stratégie communicative qu'adopte le scripteur dans son discours.

Il ne s'agit pas seulement de la simple conséquence d'une compétence dans deux ou plusieurs langues, mais d'un acte langagier qui obéit à des stratégies de communication socialement significatives. Selon Bernard Zongo (1996) : «la démonstration a été faite que l'alternance linguistique constitue une stratégie communicative et non un simple mélange linguistique aléatoire et arbitraire comme on a eu pendant longtemps tendance à le croire» (341). En ce sens, le locuteur-journaliste (que nous appellerons « locuteur collectif», en référence à «locuteur-journal»), dont l'une des fonctions est de rapporter des informations, exerce son métier dans un contexte communicationnel complexe : il est énonciateur, parle deux ou plusieurs langues et s'adresse à un public (énonciataire) qui, lui aussi, parle deux ou plusieurs langues. Ajoutons à cela le fait que les deux protagonistes ont des attitudes sociolinguistiques à l'égard de ces langues.

$5 \quad$ Le journaliste se trouve également dans une position de rapporteur des propos de ses interlocuteurs (citations, reprises de slogans, etc.). D'un point de vue sociolinguistique, cette position met le locuteur devant des choix significatifs : quelle langue utiliser dans le discours de l'autre (discours rapporté ou citation)? L'arabe dialectal est langue véhiculaire/vernaculaire, l'arabe standard est langue officielle, le berbère est langue nationale vernaculaire. Faut-il rapporter les propos d'autrui en arabe, en berbère, en français, dans une seule, deux, trois ou plusieurs langues? Faut-il les traduire ? Ce sont ces questions que se pose un journaliste francophone avant de rapporter un discours qui n'a pas été initialement produit en langue française.

Ainsi, dans ce plurilinguisme ambiant, plusieurs voix se manifestent et se confrontent. Plurilinguisme et polyphonie se conjuguent puisqu'il s'agit, pour le journalistescripteur, d'opérer des choix parmi plusieurs langues et de les distribuer sur les voix qu'il fait intervenir dans son discours.

\section{Le contexte du corpus}

7 Habituellement, l'alternance de langues est étudiée dans les cas où la langue de communication est la langue maternelle. Les chercheurs s'intéressent aux conséquences du contact de langues présentes dans une même aire géographique. L'alternance de langues qui nous intéresse dans cette étude est très particulière: le français est langue de communication puisqu'il s'agit ici de la presse francophone, l'arabe standard - la seule langue officielle - occupe des situations formelles, et l'arabe dialectal - langue orale- est langue «de grande communication et d'intercompréhension sociale forte » (Queffélec et al. 2002 : 109).

Dans le cas de l'Algérie, l'alternance de langues est étudiée dans des situations où le discours est produit en arabe ou en berbère. L'alternance codique fonctionne selon les schémas : arabe/français ou berbère/français (Morsly, 1995: 20). Le phénomène que nous étudions concerne une pratique discursive dans laquelle le locuteur écrit en français, en faisant appel aux langues nationales, à savoir l'arabe et le berbère.

Le corpus sur lequel nous avons travaillé est puisé dans quatre journaux : El Moudjahid, El Watan, La Tribune et Liberté. Ceux-ci ont des lignes éditoriales différentes inhérentes à leurs statuts. El Moudjahid est un journal national (propriété étatique). Les trois autres 
journaux appartiennent à des entreprises privées. Ce choix permet de comprendre la structuration des alternances codiques dans chaque journal.

Nous avons choisi de travailler sur des périodes très riches en actualités sociopolitiques : le mois de décembre 2006 et les mois d'avril et mai 2007 marqués par des élections sénatoriales (décembre 2006), des élections législatives (mai 2007) et des attentats terroristes (avril 2007).

11 Nous avons dépouillé 4781 articles, sélectionnés dans 158 numéros, et constitué un corpus de 88961 formes (occurrences).

Pour détecter les alternances de langues dans leurs contextes d'utilisation, nous avons eu recours à trois filtres : Word 2007, Lexico 3 et TermoStat. Cependant, nous avons dû également procéder à des dépouillements empiriques pour le pointage de certains cas d'alternances de langues spécifiques que ces logiciels, bien que très efficaces, n'ont pas détectés.

\section{Le plurilinguisme polyphonique}

13 Le recours aux langues en présence en Algérie se manifeste dans le discours médiatique à travers l'usage de l'alternance de langues. En rapportant l'information, les journalistes font intervenir plusieurs voix dans leurs discours. Ils utilisent divers procédés pour introduire des voix-témoins susceptibles de rendre l'information plus crédible. Ils recourent aux citations (discours rapportés) pour créer l'effet d'objectivité et d'authenticité. L'alternance de langues permet de rendre compte de cette altérité.

L'intégration de la voix du locuteur-témoin dans le discours du journaliste permet de tracer des frontières entre les deux discours. Le discours rapporté (voix du locuteurtémoin) est maintenu souvent dans son énonciation première : avec ou sans traduction. Cela montre un certain choix du locuteur-journaliste concernant la structuration de l'alternance codique. Ulla Tuomarla (1997) explique que "la scène énonciative journalistique se caractérise par une absence de marque du locuteur principal (le journaliste) compensée par une multitude d'autres voix que celui-ci expose » (110).

Dans le contexte algérien, ces voix qui interviennent dans le discours médiatique francophone sont plurilingues. C'est la raison pour laquelle nous parlons de plurilinguisme polyphonique.

Nous avons mentionné dans l'introduction de cet article que le journaliste algérien francophone est confronté à plusieurs choix dans son acte d'énonciation. En plus de la multiplicité des langues auxquelles il a affaire, il se trouve face au dilemme de la distribution des langues sur les voix qu'il fait parler dans son discours.

17 La polyphonie désigne "un discours où s'exprime une pluralité de voix" (Moeschler et Reboul, 1994 : 92). Selon Alexandra Kratschmer et al. (2009), ce concept renvoie au fait qu' un énoncé est susceptible de véhiculer des traces d'autres "voix", "opinions" ou "points de vue" que ceux du locuteur » (115).

18 Nous constatons que ce concept, dans la situation du discours journalistique et particulièrement dans le phénomène que nous étudions (l'alternance codique), renvoie aux multiples co-énonciateurs qui participent à l'énonciation du journaliste. 
19 En intégrant l'aspect sociolinguistique dans le concept de polyphonie, la dimension plurilingue apparaît au carrefour des choix de langue que l'énonciateur doit faire dans son acte d'énonciation. Or, le journaliste algérien est confronté à une situation sociolinguistique délicate. En effet, en Algérie, le conflit linguistique, qui est complexe à tout point de vue, concerne non seulement des langues différentes, mais aussi les mêmes langues, à cause des variétés qui s'opposent dans chaque langue.

21 Cette ambiance conflictuelle place le journaliste dans des postures qui exigent de lui des choix dans l'acte d'énonciation. Cette situation relève de la polyphonie plurilingue, puisqu'elle met la multiplicité des langues en corrélation avec la multiplicité des voix dans le discours.

Par ailleurs, la frontière entre l'énoncé assumé par le journaliste et l'énoncé rapporté est marquée par le changement de langue : français/arabe dialectal ou standard et berbère.

Dans la presse écrite, le locuteur-scripteur adopte plusieurs positions énonciatives. Ces positions sont soumises à des contraintes liées au contexte par ce que P. Charaudeau (2000) désigne à travers la notion de « contrat médiatique » ${ }^{65}$.

En effet, l'énonciateur-journaliste subit trois types de pressions qui déterminent son acte de parole (acte d'écriture) : l'instance médiatique (entreprise, son recruteur qui impose une ligne éditoriale), l'instance professionnelle (les exigences du métier de journaliste : objectivité, éthique et déontologie), l'instance commerciale (représentée par le consommateur qui attend du journaliste une information). Le journaliste obéit donc à un règlement imposé par son employeur (le journal), respecte l'éthique imposée par son métier et prend en considération l'horizon d'attente du lecteur.

\section{La structuration de l'éenonciation dans les alternances de langues}

L'alternance de langues apparaît parmi les choix linguistiques que le journaliste doit opérer dans son discours. Ceux-ci reflètent l'attitude du locuteur envers les langues.

Trois postures permettent de situer l'alternance des langues dans notre corpus. La première est exprimée par le changement de langues: français/langues d'Algérie (arabe ou berbère). La deuxième concerne les situations dans lesquelles le discours rapporté est exprimé selon le schéma : français/langues d'Algérie (arabe et berbère) + français. La troisième posture apparaît lorsque l'énonciateur-scripteur est confronté à l'alternance des langues et où il alterne lui-même les langues avec ou sans le recours à la citation. 


\section{La première posture}

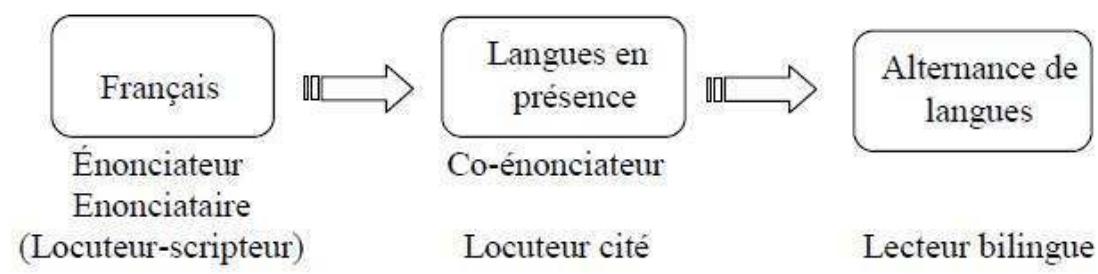
co-énonciateur dans la langue de sa production d'origine : ici l'arabe dialectal, l'arabe standard ou le berbère. Le journaliste-scripteur est totalement absent de l'énonciation de son interlocuteur. Les guillemets marquent la rupture entre les deux énonciateurs. L'effacement énonciatif est motivé par la recherche de l'objectivité, de la neutralité et de la précision : c'est l'enjeu de la crédibilité, selon Charaudeau P. (2006). L'effacement énonciatif constitue dans ce cas une stratégie "permettant au locuteur de donner l'impression qu'il se retire de l'énonciation, qu'il "objectivise" son discours » (Vion, 2001 : 210). Le journaliste fait l'effort de gommer toute marque qui permette d'identifier la source de l'énoncé :

(9) El Watan : Un homme vient de voter. Désabusé, il nous lance d'un ton malicieux (discours du journaliste produit en français): «El meyyet khella tarka wel waratha kesmouha » (discours du co-énonciateur produit en arabe dialectal) ("le mort a laissé un héritage et les héritiers se sont partagés la succession") (discours traducteur du journaliste produit en français)

(18) Liberté : Ils sont en mesure de le prouver en toute circonstance. "Maadnach etben fi karchna " ( "il n'y a pas de paille dans notre ventre"), a-t-il ajouté à plusieurs reprises.

(42) La Tribune : (...) par des potaches à l'air soucieux. « Ma t'hallitch fiya », lance un postulant à un autre (...).

Dans les exemples (9) et (18) la phrase introductive est énoncée en français. La rupture entre les deux discours, celui du journaliste et celui du locuteur-témoin, est marquée par les guillemets. L'arabe dialectal est attribué à la personne citée. Le journaliste se réapproprie également la voix de son interlocuteur pour traduire son discours, faisant ainsi apparaître la fonction de réitération de l'alternance des langues. Dans l'exemple (42), le locuteur-scripteur ne traduit pas le discours de son interlocuteur. L'énonciataire est supposé connaître les deux langues.

\section{La deuxième posture}

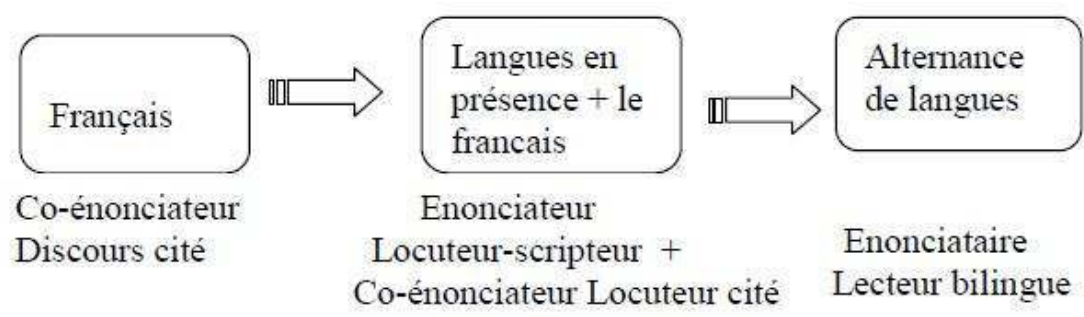

29

À travers cette posture, on constate que le journaliste trace graphiquement (par l'emploi des guillemets) la frontière entre ses propres propos et le discours de son co-énonciateur. Mais, il peut se manifester aussi dans le discours de son co-énonciateur 
en le traduisant en français. Dans certains cas, il maintient quelques expressions dans la langue de leur production. L'effacement énonciatif est maintenu car le journaliste est absent dans le propos énoncé. Dans les exemples 1 (El Watan) et 11 (La Tribune), la voix de l'énonciateur et celle du co-énonciateur alternent et se mélangent. L'énonciateur est présent seulement par sa traduction du discours rapporté. Le co-énonciateur est présent par un double jeu de voix : il est présent, d'une part, car il est cité (les verbes introducteurs annoncent sa participation dans le discours : "interroge un citoyen », " a relevé $M$. Ouyahia »), et d'autre part, par la langue et le contenu de la prise de parole : langues d'Algérie et contenu du discours.

La structuration de ce type d'alternance est particulière puisque le locuteur-scripteur alterne, à l'intérieur du discours cité, les langues en présence et le français. Il sélectionne ce qu'il faut maintenir dans la langue d'origine et ce qu'il faut énoncer en français :

(1) El Watan: «Bentalha yekhedmou biha (Voix du co-énonciateur: en arabe dialectal). Bentalha leur sert d'alibi pour appâter l'opinion internationale (Voix énonciateur/co-énonciateur : réitération en français). Où est passé tout l'argent qu'ils ont reçu sur notre dos depuis 1997?», interroge un citoyen (Voix énonciateur/co-énonciateur : discours rapporté en français).

(7) El Watan: J'ai voté à blanc parce qu'aucun de ces candidats ne me convainc. Aucun d'eux n'a l'honnêteté de nous dire en face, à la télé: ma andi ma ndirlkoum,( je ne peux rien pour vous). Tous te promettent le Paradis, après, ils t'abandonnent dans ton enfer ! », tranche-t-il.

(11) La Tribune : Il fallait tenir bon et ne rien céder, c'est ce que nous avons fait et el hamdoulillah, nous sommes arrivés à ces résultats, c'est la victoire des travailleurs et celle du complexe.

31 Dans l'exemple 1 (El Watan), le journaliste enclenche la parole de son co-énonciateur en arabe dialectal. Le même énoncé est produit ensuite en français sans aucune mention, ni métalinguistique, ni graphique. Le discours rapporté se poursuit en français, laissant supposer que le journaliste a lui-même pris en charge la parole de son interlocuteur, en décidant des segments à prononcer en arabe et de ceux en français. Le même constat s'impose pour l'exemple 7 (El Watan).

\section{La troisième posture}
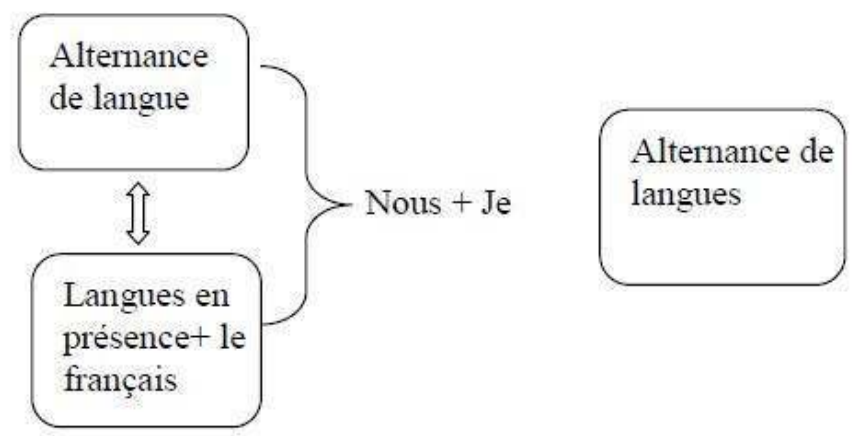
Enonciateur Locuteur-scripteur +
Co-énonciateur Locuteur cité
Enonciataire

Lecteur bilingue

Le journaliste participe à la construction de son énonciation et manifeste sa présence dans le discours en prenant position par rapport à son dire. C'est ce que nous 
constatons à travers les exemples 11 (El Watan), 14 (El Moudjahid), 26 (La Tribune) et 28 (La Tribune).

Ainsi, l'énonciateur pratique lui-même l'alternance codique : il prend en charge l'acte d'énonciation et introduit les langues en présence dans son discours. La citation se manifeste implicitement dans son discours.

Dans la séquence 11 (El Watan), le commentaire est formulé à travers une expression populaire. Le journaliste qui a énoncé la formule émet un commentaire métalinguistique pour expliquer le contenu de son discours : «Cette belle formule puisée dans le génie populaire algérien, intraduisible dans la langue de Molière, résume parfaitement l'état d'esprit des citoyens de la capitale en ce jeudi électoral».

C'est le même constat pour l'extrait 27 (La Tribune) dans lequel le journaliste emploie l'arabe dialectal pour commenter un événement (hypothétique): l'éventualité de l'élection d'une femme à la tête du pays :

(11) El Watan : Pour le moment, c'est toujours « recham H'mida, l'âab H'mida !" Cette belle formule puisée dans le génie populaire algérien, intraduisible dans la langue de Molière, résume parfaitement l'état d'esprit des citoyens de la capitale en ce jeudi électoral.

(14) El Moudjahid: Les Jordaniens d'El-Fayçali ont adopté une position tout à fait aux antipodes de celle des gars de "Chibh El-Djazira El-Arabia").

(26) La Tribune : Une femme ne devrait pas gouverner le pays, mais si c'est Louisa " maâlihch ".

(28) La Tribune: Endiguer aussi la malversation et les déviations à travers l'initiative «fassad qif» (stop corruption), qui ne vise pas seulement le côté économique.

Dans l'exemple 14 (El Moudjahid), l'arabe standard est cité pour nommer l'appartenance géographique d'une équipe de football. Mais cette citation n'est pas attribuée. Elle est assumée par le journaliste.

Dans l'exemple 28 ( $L a$ Tribune), l'arabe standard apparaît à travers un slogan gouvernemental contre la corruption. Le journaliste alterne les langues sans attribuer ce discours à une source définie.

Dans les exemples 11 (El Watan) et 27 (La Tribune), l'arabe dialectal apparaît à travers des commentaires énoncés par le journaliste lui-même.

\section{Les grandes tendances de l'alternance de langues}

Nous avons distingué trois tendances de l'alternance des langues: l'utilisation des expressions figées à travers lesquelles l'arabe dialectal est employé comme expression polyphonique, le recours aux savoirs partagés (formules d'invocation de Dieu et du serment), et enfin le recours aux proverbes et aux adages populaires.

Les expressions figées sont un élément de l'interdiscours. Ce terme emprunté à Anne Salazar Orvig (1999) désigne la sphère de l'échange culturel regroupant des «savoirs partagés ». Il se manifeste à travers l'écrit journalistique sous diverses formes: les savoirs partagés, les proverbes et les détournements. Les expressions figées repérées dans notre corpus ont une double fonction : argumentation et expression d'implicites culturels (ironie, insinuation et métaphore).

41 Les savoirs partagés convoquent des savoirs génériques : religieux et culturels. Les cas d'alternances de langues dans notre corpus renvoient à l'emploi de formules 
d'invocation de Dieu et de serment et à des expressions populaires figées que seuls les membres initiés à une même culture peuvent comprendre. Ces expressions révèlent une intention de connotation culturelle.

Elles apparaissent dans notre corpus souvent en arabe dialectal. Dans la presse francophone algérienne, elles se manifestent à travers deux voix: le journaliste (locuteur-scripteur) et le locuteur cité. Le locuteur-scripteur fait appel à ces formules qui fonctionnent comme des marques identitaires. Dans les extraits 8 (El Moudjahid) et 25 ( La Tribune), les formules d'invocation de Dieu sont employées dans les séquences de fermeture. Elles sont constitutives des conventions de communication propres aux locuteurs musulmans arabophones: invoquer Dieu signifie, le plus souvent, être musulman et croire en sa puissance et en son omnipotence. Le journaliste qui écrit en français en parlant d'un futur que seul Dieu commande et connait, invoque Dieu en arabe: inchallah (Si Dieu veut). Cette expression est entrée dans les habitudes langagières des Algériens et son emploi s'étend même aux personnes non musulmanes.

Mais il faut noter que cette alternance n'est pas omniprésente dans les écrits attribués en propre aux journalistes. Dans notre corpus, nous n'en avons relevé que très peu. Elles apparaissent chez des co-énonciateurs que le journaliste cite dans son discours. Il s'agit de la deuxième voix prêtée à un troisième interlocuteur qui intervient dans le discours comme co-énonciateur.

Le locuteur-scripteur rapporte le discours de l'autre en utilisant l'arabe dialectal, puis traduit le reste du discours en français. Ce procédé est intéressant à analyser parce qu'il rend compte d'une caractéristique spécifique au discours des Algériens.

En effet, le journaliste opère un choix dans la parole de son interlocuteur. En maintenant les formules d'invocation divine en arabe dialectal et en traduisant le reste du discours en français, il opte pour l'utilisation simultanée de deux langues. Le discours initial est produit en arabe dialectal. Les formules d'invocation de Dieu et du serment sont énoncées en arabe dialectal. Cette récurrence dans l'emploi de l'alternance codique dans le discours est motivée par la valeur que les interlocuteurs accordent à la relation qui s'établit entre eux: locuteur/énoncé, locuteur/ co-énonciateur, locuteur/ lecteur.

Dans toutes les séquences du corpus, où les langues sont alternées, les formules d'invocation de Dieu sont employées dans les types de séquences suivantes :

- évocation du futur (connu de Dieu seul) : inchallah (Si Dieu veut)

- satisfaction (réalisée grâce à Dieu) : elhamdoulillah (Dieu soit loué)

- invocation (procédé de crédibilisation de la parole) : ouallah (Je jure par Dieu),

- résignation : Allah ghalab (A Dieu ne plaise).

Les invocations de Dieu interviennent sous forme de séquences de fermeture de la parole quand il s'agit du futur. Elles apparaissent également dans les ouvertures de la parole dans la formule du serment (Ouallah). Les autres formules d'invocation de Dieu fonctionnent avec la même configuration: le journaliste, en donnant la parole à un interlocuteur, glisse du français à l'arabe pour invoquer Dieu, puis reprend son discours en français.

L'alternance se réalise selon le schéma suivant: français//arabe//français. Les formules d'invocation de Dieu fonctionnent comme des citations puisqu'elles sont épinglées entre les guillemets. Dans ce cas de figure, le passage d'une langue à une autre est dicté par la relation qu'établit le journaliste avec ses lecteurs : il s'agit d'un 
marquage identitaire. Cela obéit à un ordre de discours co-construit par tous les acteurs du même discours. Kerbrat-Orecchioni (1996) parle d'un discours qui obéit à la relation de dépendance conditionnelle. Ce sont donc des expressions idiomatiques auxquelles le groupe est attaché. Ce type d'expressions installe une connivence (Melliani, 2000) avec les membres du groupe.

Par ailleurs, dans La Tribune, l'expression "h'na imout Kaci», littéralement « ici meurt Kaci », est une expression populaire algérienne qui exprime l'entêtement quand il s'agit de vouloir se maintenir à un endroit ou de camper sur ses positions. "Kaci » fait référence à une légende dans laquelle ce prénom désignait un personnage de la région de Kabylie connu pour la fermeté de son entêtement.

Dans un extrait d'El Watan, le locuteur utilise des expressions qui convoquent des savoirs culturels partagés, intraduisibles en français, tout en sauvegardant le sens, la connotation et la même force: "H'na nefriwha, la el marikane oua la ammar bouzouar " (C'est nous qui relèverons ce pays, pas les Américains), "Rana h'na », (On est là). Ces expressions sont caractéristiques du langage algérien.

51 Les salutations, contrairement aux formules d'invocation de Dieu, ne présentent que quelques occurrences attribuées au co-énonciateur. Ces expressions, qui sont considérées comme des séquences liminaires à fonction phatique (Kerbrat-Orecchioni, 2001), ne sont que très rarement employées. Seules deux occurrences sont employées dans El Watan: «marhaban bikoum, salam alikoum». Puisqu'il n'y a pas d'interaction directe (absence d'échange langagier), les journalistes éliminent ces expressions, et ce, même dans le discours rapporté.

Les proverbes et adages populaires prennent une place importante dans notre corpus. À l'exception du quotidien El Moudjahid, les journaux recourent souvent, dans leurs discours, à des adages, proverbes et maximes en arabe dialectal.

Ces expressions figées sont repérables dans le discours rapporté ou dans le discours direct du journaliste. Elles sont utilisées comme des arguments d'autorité renforçant l'énonciation par les valeurs communes qui fondent les normes d'une société. Dans l'exemple ci-après, le journaliste ajoute un commentaire sur l'expression figée : «Pour le moment, c'est toujours "recham H'mida, l'âab H'mida!" Cette formule, puisée dans le génie populaire algérien, intraduisible dans la langue de Molière, résume parfaitement l'état d'esprit des citoyens de la capitale en ce jeudi électoral » (El Watan).

54 D’après Dominique Maingueneau (2005: 149), « le proverbe est une assertion sur la manière dont va le monde, il dit le vrai ». Le locuteur fait appel à ces expressions en utilisant l'arabe dialectal, car elles expriment une forme de «sagesse des Nations " (ibid. : 148). En mobilisant la mémoire collective, le journaliste implique ses interlocuteurs en les prenant à témoin.

55 Le journaliste d'El Watan, dans l'exemple 11 (El Watan), commentant une journée d'élection, cite l'expression populaire "recham H'mida, l'âab H'mida», qui est l'équivalent de l'expression française « être juge et partie ».

56 Le proverbe apparait comme un argument de valeur négative, extérieur à lui-même. Il fonctionne comme une sorte de repère pragmatique faisant argument (argument d'autorité), à partir d'une "évidentialité collective» (Mochet, 2003). C'est donc un argument d'autorité que le journaliste reconnaît en tant que tel avant d'en faire « usage » avec ses locuteurs. Il s'agit de dramatiser une situation à travers un énoncé collectif. Le proverbe mentionné accentue la tonalité critique de l'écrit journalistique. 
57 Si le locuteur assume ce proverbe, en revanche, il se place en opposition par rapport à la situation à laquelle ce dernier s'applique. La majorité des proverbes que nous avons relevés dans les journaux privés renvoie à des situations sociopolitiques critiquées par les journalistes ou la population : "El meyyet khella tarka wel waratha kesmouha » (le mort a laissé un héritage est et les héritiers se sont partagés la succession); «tagh ala men tagh » (c'est au plus fort) ; "Khoub'z eddar, madabina yeklou ould eddar » (il est préférable que le pain de la maison soit mangé par le fils de la maison).

Ce type d'expressions figées est supposé transmettre une vérité partagée a priori de tous, principalement le lecteur. Le proverbe renvoie à la préexistence d'un discours connu, à une intertextualité de forme et de contenu.

59 S'agissant enfin des slogans, il convient de préciser que contrairement au proverbe, le slogan est lié à la suggestion. Maingueneau (Ibid.) explique que le slogan « est avant tout destiné à fixer dans la mémoire des consommateurs potentiels l'association d'une marque et d'un argument d'achat » (149). Dans le cas de l'alternance, le slogan n'a pas pour fonction de faire vendre quelque chose, mais il remplit, par sa brièveté et sa morphologie une fonction expressive. Le journaliste cite des slogans qu'il ne fabrique pas lui-même. Ce sont généralement des slogans politiques ou syndicaux repris tels qu'ils sont produits : en arabe dialectal ou en arabe standard.

Nous avons relevé deux types de slogans : les slogans politiques et les slogans ludiques. Les slogans politiques sont repris des manifestations organisées par des partis politiques ou des syndicats. Ils sont produits soit en arabe standard soit en arabe dialectal. L'analyse des slogans collectés dans notre corpus a permis d'établir deux constats significatifs :

- les slogans officiels sont reproduits en arabe standard même dans la presse privée : (33) El Watan : (...) À l'entrée, une affiche annonce : « Haï el mouattine al nadhif » (le quartier du citoyen propre). (49) Curieusement, le slogan de ce référendum est le même que celui des législatives du 17 mai 2007: "Min ajli El Djazaïr » (Pour l'Algérie).

- les slogans populaires sont énoncés en arabe dialectal :

(8) El Watan: Un chef de centre et un chef de bureau sont payés, eux, à 4000 DA, nous dit-on. Assimilant la fonction d'encadrement électoral à du «tachghil echab ", l'emploi de jeunes.

61 Ainsi, nous pouvons avancer que l'alternance des langues, telle qu'elle se manifeste dans les journaux francophones algériens, obéit à des stratégies discursives mises en place par les journalistes afin de créer un univers communicationnel spécifiquement algérien. Le choix de telle ou telle langue dans l'alternance, la reformulation dans une langue autre que le français et l'usage des expressions idiomatiques obéissent à des mécanismes discursifs spécifiques aux journalistes algériens: il s'agit pour eux de rédiger dans un français à la fois normé et proprement local. Le choix privilégié de l'arabe dialectal dans les alternances codiques, par les journalistes, qui écrivent dans les journaux privés, est motivé par des contraintes d'ordre sociolinguistique : en employant/citant l'arabe dialectal, les locuteurs affichent une attitude positive envers cette langue.

63 L'alternance à laquelle nous avons affaire traduit la configuration réelle du fonctionnement des langues en Algérie : l'arabe standard et le berbère sont confinés dans des usages restreints. 


\section{BIBLIOGRAPHIE}

CHARAUDEAU P., « Discours journalistique et positionnements énonciatifs, Frontières et dérives », Semen, 22, Énonciation et responsabilité dans les médias, 2006. Disponible sur le site [https:// journals.openedition.org/semen/2793].

CHARAUDEAU P., « « L'événement dans le contrat médiatique », in Dossiers de l'audiovisuel, n 1 , « La télévision de l'événement », La documentation française, Paris, mai-juin, 2000. Consultable sur le site : http://www.patrick-charaudeau.com/L-evenement-dans-le-contrat.html

KERBRAT-ORECCHIONI C., La conversation, Paris, Seuil, 1996.

KERBRAT-ORECCHIONI C., Les actes du langage dans le discours, théorie et fonctionnement, Paris, Nathan Université, 2001.

KRATSCHMER A., BIRKELUND M., THERKELSEN R., La polyphonie : outil heuristique linguistique, littéraire et culturel, Berlin, ED. Frank \& Timme GmbH Verlag für wissenschaftliche Literatur, 2009.

MAINGUENEAU D., Analyser les textes de communication, A.Colin, 2005.

MORSLY D., «L'alternance des codes dans la conversation des locuteurs algériens ", in Véronique D., Vion R. (éds), Des savoirs communicationnels, Université Provence, 1995, pp. 19-29.

MELLIANI F., La langue du Quartier : Appropriation de l'espace et identités urbaines chez des jeunes issus de l'immigration maghrébine en banlieue rouennaise, L'Harmattan, 2000.

MOCHET A., « Mention et/ou usage : discours direct et discours indirect libre en situation de type conversationnel », in Authier-Revuz J., Doury M., Reboul-Touré S. Parler des mots. Le fait autonymique en discours, Paris : Presses de la Sorbonne Nouvelle, 2003, pp. 161-174.

MOESCHLER J. et REBOUL A., « Polyphonie et énonciation », Dictionnaire encyclopédique de pragmatique, Paris, Seuil, 1994.

QUEFFÉLEC A. et al., Le français en Algérie. Lexique et dynamique des langues, Louvain-la-Neuve, Duculot-AUF, 2002.

SALAZAR-ORVIG A., Les mouvements du discours. Style, référence et dialogue dans des entretiens cliniques, Paris, L'Harmattan, 1999.

TUOMARLA U., « Sur le fonctionnement discursif de la citation », in Välikangas \& Härmä (éds), Où va le français ?, De Werelt, Ámsterdam,1997, pp. 109-116.

Vion R.,"Modalités, modalisations et activités langagières", Marges Linguistiques, nº 2, M.L.M.S. éd., 2001, pp. 209-234.

zONGO B., « Alternance des langues et stratégies langagières en milieu d'hétérogénéité culturelle : vers un modèle d'analyse ", in Caroline Juillard \& Louis-Jean Calvet (dir.), Les Politiques linguistiques, mythes et réalité, Beyrouth, AUPELF-UREF, 1996, pp. 341-349.

\section{NOTES}

65. Pour Patrick Charaudeau, «Toute situation de communication (...) constitue un cadre de contraintes dont devront tenir compte les partenaires de l'échange faute de quoi il n'y aurait pas d'intercompréhension possible. Ces partenaires se trouvent donc dans une relation de réciprocité 
et de reconnaissance de la finalité de leur situation de communication, de leur identité, du propos qu'ils échangent et des circonstances dans lesquelles ils se trouvent, raison pour laquelle je propose la notion de «contrat de communication » pour expliquer le phénomène de la communication sociale ", "L'événement dans le contrat médiatique », in Dossiers de l'audiovisuel, $\mathrm{n}^{\circ} 1$, « La télévision de l'événement », La documentation française, Paris, mai-juin, 2000, consulté le 30 avril 2013 sur le site de Patrick Charaudeau - Livres, articles, publications.

URL : http://www.patrick-charaudeau.com/L-evenement-dans-le-contrat.html

\section{RÉSUMÉS}

Le français, tel que parlé et écrit en Algérie, présente des variations lexicales importantes qui nous ont amené à nous interroger sur quelques-unes de ses caractéristiques dans la presse écrite algérienne. Nous nous sommes précisément intéressés à l'alternance codique et aux mécanismes discursivo-argumentatifs qui participent au façonnement du français employé dans quatre journaux francophones, à savoir El Moudjahid, El Watan, La Tribune et Liberté. L'analyse du corpus a révélé que l'alternance des langues arabe, berbère et française, telle qu'elle se manifeste dans ces quatre quotidiens, obéit à des stratégies discursives mises en place par les journalistes pour créer un univers communicationnel spécifiquement algérien.

French, as it is spoken and read in Algeria, presents very important lexical variations that led us to inquire about some of these characteristics in the Algerian written press. We are more precisely interested in code switching and the discursivo-argumentative mechanisms which take part in modeling the French language employed in four newspapers namely, El Moudjahid, El Watan, La Tribune, Liberté. Analysis of the corpus reveals that the alternation of the Arabic, Berber and French languages, as it appears in these four daily newspapers, obeys to discursive strategies used by journalists in order to create a specifically Algerian communicational universe.

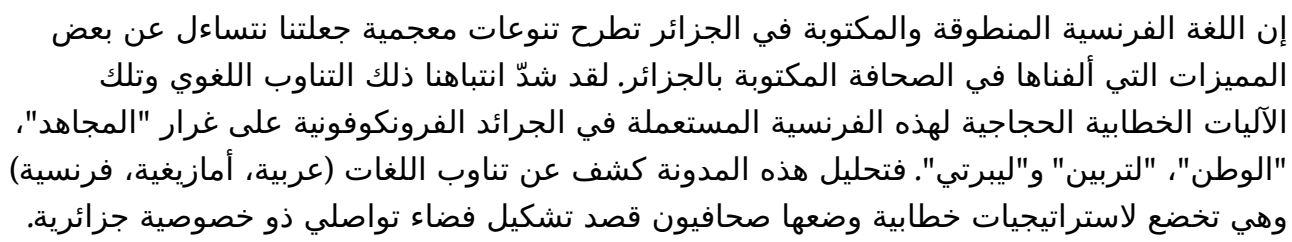

\section{INDEX}

Keywords : code switching, multilingualism, lexical particularisms, lexical variation, algerian francophone press

$$
\text { التناوب اللغوي, التعدد اللغوي, الخصائص المعجمية- التنوعاتفهرس الكلمات المفتاحية: }
$$

Mots-clés : alternances de langues, plurilinguisme, particularismes lexicaux, variation lexicale, presse francophone algérienne 
AUTEUR

MOURAD BEKTACHE

Université A. Mira - Laboratoire LAILEMM - Bejaia - Algérie 